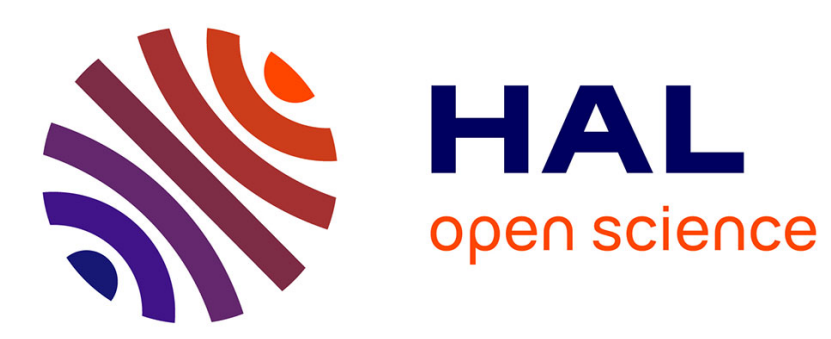

\title{
Thin Films and Fine Powders of Ferrites: Materials for Magneto-Optical Recording Media
}

\author{
P. Tailhades, Louis Bouet, L. Presmanes, A. Rousset
}

\section{To cite this version:}

P. Tailhades, Louis Bouet, L. Presmanes, A. Rousset. Thin Films and Fine Powders of Ferrites: Materials for Magneto-Optical Recording Media. Journal de Physique IV Proceedings, 1997, 07 (C1), pp.C1-691-C1-694. 10.1051/jp4:19971283 . jpa-00254996

\section{HAL Id: jpa-00254996 https://hal.science/jpa-00254996}

Submitted on 1 Jan 1997

HAL is a multi-disciplinary open access archive for the deposit and dissemination of scientific research documents, whether they are published or not. The documents may come from teaching and research institutions in France or abroad, or from public or private research centers.
L'archive ouverte pluridisciplinaire HAL, est destinée au dépôt et à la diffusion de documents scientifiques de niveau recherche, publiés ou non, émanant des établissements d'enseignement et de recherche français ou étrangers, des laboratoires publics ou privés. 


\title{
Thin Films and Fine Powders of Ferrites: Materials for Magneto-Optical Recording Media
}

\author{
P. Tailhades, L. Bouet, L. Presmanes and A. Rousset
}

LCMI, URA 1311 du CNRS, Université Paul Sabatier, 31062 Toulouse, France

\begin{abstract}
In the near future, the magneto-optical (MO) recording will require materials having high MO effects at short wavelengths (400-500 nm). Ferrites, especially garnets, but also spinel ferrites have highly advantageous MO properties in this spectrum and also have very stable structural and chemical properties. The present paper evaluates their used as MO media, in comparison with the rare-earth transition metals alloys and the metallic multilayers.
\end{abstract}

\section{INTRODUCTION}

Magnetic, magneto-optical (MO), phase change recordings and the silicon "flash" memories are the four reversible recording technologies currently competing on the market. The importance of each in the future will depend on their ability to record high density information, to access the informations in very short times, and to provide low cost reliable media and drives.

For the time being, magnetic storage satisfies the above requirements quite well, is the most widely used of the four previous technologies and this strong position is reinforced by continuous and rapid improvements in its performance. For instance, the storage density of hard disks is currently doubling every eighteen months. To record a bit, a magnetic head can rub against the storage medium (as in magnetic tapes) or fly over it (hard disk). The latter process allows very short access times. With it, future increase in recording density will require a decrease in head-medium gap. To day, this gap is of the order of several tens of nanometers in hard disk drives, which makes hard disks unremovable media. MO heads, on the other hand, fly over the media at more than one millimeter, to write and read high density information. Consequently, the MO technique offers short access time and high density for removable media. These characteristics make MO recording the number one competitor of the magnetic technology. Moreover, computer 3"1/2 and 5"1/4 disks and drives, and especially, the audio $2^{\prime \prime} 1 / 2$ mini-disks and drives, are very large markets for the development of the MO technique.

The basic principle of the phase change writing on ternary Ge-Te-Sb alloys is to create a sequence of amorphous and crystalline zones, by proper laser heating. To read the bits, the amorphous and the crystalline zones are discriminated by their different reflectivities. In MO detection, on the other hand, the beam reflected by a magnetized zone has to be analysed. Phase change recording therefore requires a simpler optical detection than MO drives. This simplicity is the main advantage of phase change recording. Unfortunately, phase change media generally do not have very good cyclability.

Silicon memories are mainly attractive because of their very short access time. However, while their capacity is now quite good (64 Mbytes), their price per megabyte (\#1\$) is still too high.

Ferrites would help increase the performance of magnetic and MO recordings, by improving the media properties. In fact, the magnetic recording on hard disks will have to improve the storage density by decreasing the gap between the head and the magnetic layer. In the near future, it will thus be necessary to remove the protective film deposited on the cobalt alloys recording layers. This would bring a gain of about ten nanometers. However, current cobalt alloy recording films would have to be replaced by ferrimagnetic oxides, as these are not sensitive to corrosion. Much research is currently being done on barium and spinel ferrites in this direction. MO recording density is also currently limited by the diffraction phenomenon, which makes the minimum readable bit size close to $0.5 \mathrm{~N}$ NA ( $\lambda$ being wavelength of the laser spot and NA the numerical aperture of the objective lens). Any increase in MO storage density thus requires shorter laser wavelengths or optical systems having a high numerical aperture. For the time being, this aperture seems to be limited to about 0.6 . Current laser diodes with sufficient power and reliability to be used for recording applications have wavelengths of 780 or $680 \mathrm{~nm}$. In a near future, shorter wavelengths might become possible using new laser diodes or frequency doubling systems. However, MO recording with short wavelengths will require materials having sufficiently high Faraday or Kerr effects in this spectrum. The amorphous rare earthtransition metal alloys (RE-TM) currently used as storage materials for all the commercially available media do not exhibit 
strong MO effects for these radiations. The ferrites, especially gamets and spinels, have better MO properties, and also offer very good corrosion resistance and a high level of crystallographic stability.

The outlook for ferrites in both magnetic and MO recording could be discussed on the basis of the above analysis; but this paper is limited of the possible use of ferrites, especially spinel ferrites, in future MO media. The main properties required for MO recording materials are first briefly reviewed, and then the drawbacks and the advantages of the most promising $\mathrm{MO}$ materials, followed by a discussion of possible ways of solving current problems encountered in applying ferrites to MO media.

\section{MAGNETO-OPTICAL RECORDING MATERIAL REQUIREMENTS}

In writing an MO bit, a small spot of the recording layer is heated by means of a high power (about $10 \mathrm{~mW}$ ) laser beam to above its Curie temperature. The bit value 0 or 1 is then determined by the direction (up or down) of a magnetic field applied during the heating. In read mode, the spot is illuminated by a low power (about 1-2 $\mathrm{mW}$ ) linearly polarised laser beam. The 0 or 1 value is detected by the rotation of the polarisation plane, which is rotated positively or negatively depending on whether the analysed region is magnetized up or down. Therefore, the $\mathrm{MO}$ material must have a Curie point not too far above room temperature $\left(200-300^{\circ} \mathrm{C}\right)$, to be able to write with a reasonable laser power; a sufficiently high Faraday or Kerr rotation; a strong perpendicular anisotropy; and a high transparency or reflectivity for reading. Moreover, a strong coercivity ( $>1000-2000$ Oe) is necessary, to stabilize small bits; low roughness, high homogeneity, high corrosion resistance; a good structural stability of finely grained or amorphous films is also required to prevent media noise and to ensure sufficient recorded data life-time.

Three main families of materials potentially offer most of the above requirements and also have strong assets of use as short-wavelength recording media: TM-RE amorphous alloys, multilayered metallic structure and ferrites.

\section{AMORPHOUS RARE EARTH-TRANSITION METAL ALLOYS.}

In this class of materials, certain alloys containing Tb-Fe-Co, Gd-Tb-Fe, Dy-Fe-Co, among others, meet most of the above requirements. Besides their high coercivity and $\mathrm{MO}$ effects for the red wavelengths and their strong perpendicular anisotropy, the RE-TM alloys also have a very smooth surface and no grain boundaries because of their amorphous structure. These latter two characteristics are very important in minimizing the media noise. Moreover, amorphous RE-TM alloys are obtained directly by room temperature sputtering, without post-annealing treatments, which allows the use of cheap polycarbonate substrates. The high reactivity to oxygen of not only the rare earths but also the transition metals makes these alloys unstable in air. However, in MO media these materials are sandwiched between dielectric layers, such as $\mathrm{AlN}_{\mathrm{N}}$ or $\mathrm{Si}_{3} \mathrm{~N}_{4}$, to prevent corrosion and enhance the MO effect by interference phenomena. This can alleviate the oxidation damage, but a structural relaxation and a crystallization (inherent to the amorphous structure of the RE-TM alloys [1][2]) may also occur during the write-read-erase cycles. It seems that structural relaxation will significantly modify the magnetic properties after just a year, and crystallization will do the same after a decade [3]. These drawbacks have not yet been eliminated; but for the time being they are acceptable for practical recording applications. Currently, all commercially available MO media have a recording layer made of RE-TM amorphous alloys.

The amorphous RE-TM alloys generally have lower Kerr rotation and reflectivity in the green or blue spectra than in the red. These are the main drawbacks for their future applications in MO media. Certain Pr-Fe-Co or Nd-Fe-Co amorphous alloys with high Kerr rotations for blue radiations [4], have already been found, but their low Curie temperature is incompatible with the recording requirements. Moreover, only a marginal gain in MO media performance was observed by incorporating $\mathrm{NdFeCO}$ alloys into optimized TbFeCo thin films stacks [5]

\section{MULTILAYERED METALLIC STRUCTURES.}

Multilayers of very thin films (less than 1 or $2 \mathrm{~nm}$ ) of Co/noble metal [6][7][8], especially Co/Pt and Co-Ni/Pt stackings, are potential candidates for $\mathrm{MO}$ recording applications with blue radiation. In this spectrum they have higher Kerr effects than those of the RE-TM alloys, and are more stable against oxidation because of the presence of the noble metal. These two characteristics are the main advantages of the multilayered structures.

$\mathrm{The} \mathrm{Co} / \mathrm{Pt}$ or $\mathrm{Co}-\mathrm{Ni} / \mathrm{Pt}$ multilayers are polycrystalline. Their coercivity is thus strongly dependent on their microstructure. Both high coercivity and small grain sizes are difficult to obtain in these materials. Because low crystallization is required in order to prevent media noise, much smaller coercivities are reached in practise, compared to amorphous RE-TM alloys. Lower stability of the small stored bits is then obtained. The high Curie temperature of the Co/Pt, which is responsible of a rather low writing sensitivity, can be reduced by alloying cobalt with nickel [9]. The writing sensitivity can also be improved using a seed layer of mixed tin and indium oxide [10]. However, because of the ferromagnetic order, high saturation magnetization tends to form subdomains inside the bits stored in Co/noble metal multilayers. The subdomains can contribute to the noise in read mode. Interdiffusion between the two components of the structure can also be a drawback for these materials, which can lead to a gradual loss of the initial magnetic properties. In spite of the potential drawbacks mentioned above, MO media have been manufactured with good performance [11] even in blue spectrum. Low cost mass production of homogeneous stackings of very thin films seems to be difficult however, especially for large disk formats. 


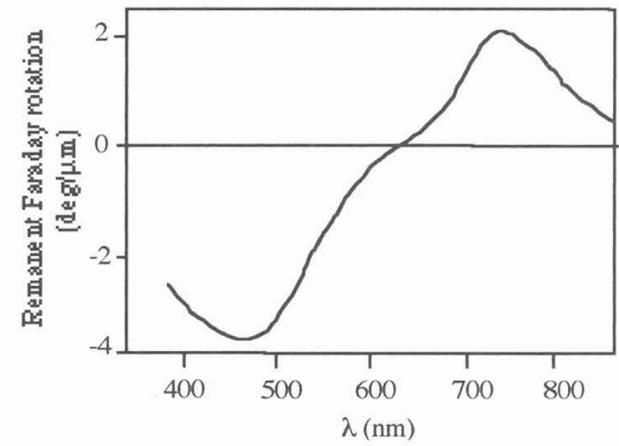

Figure 1: Remanent Faraday rotation versus wavelength for a thin film

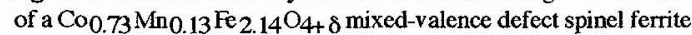

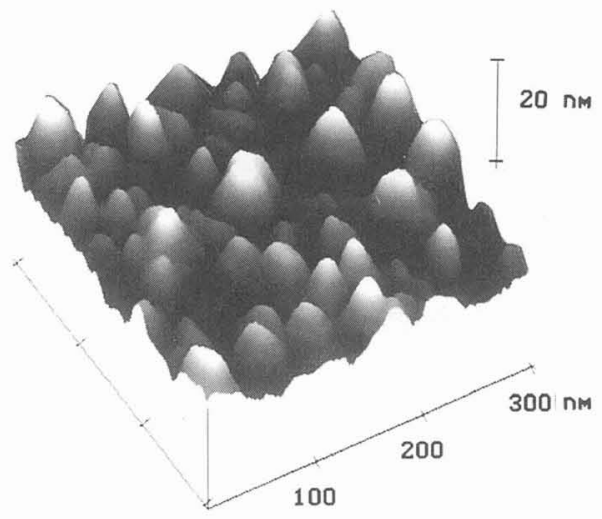

Figure 2: AFM micrograph of a Co-Mn spinel ferrite film annealed at $450^{\circ} \mathrm{C}$.

\section{FERRITES.}

Ferrites are of special interest because of their very high thermal stability, in contrast to RE-TM amorphous alloys and metallic multilayers. Among the ferrites, garnets films were the ones most studied for MO recording application. The most promising garnets $\left(\mathrm{Dy}_{3-\mathrm{x}} \mathrm{Bi}_{\mathrm{x}} \mathrm{Fe}_{5-\mathrm{y}} \mathrm{Ga}_{\mathrm{y}} \mathrm{O}_{12}\right.$ and $\mathrm{Gd}_{3-\mathrm{x}} \mathrm{Bi}_{\mathrm{x}} \mathrm{Fe}_{5-\mathrm{y}} \mathrm{Ga}_{\mathrm{y}} \mathrm{O}_{12}, 0.6<\mathrm{x}<1.5,0.4<\mathrm{y}<1.4$ ) have very high Faraday rotation and relatively low optical absorption at short wavelengths. Figures of merit several times higher than those of the RE-TM alloys have thus been observed [10]. Garnets films can also have stress-induced perpendicular anisotropy and quite high coercivity. The main drawbacks of garnets films stem from their polycrystalline microstructure and their high manufacturing temperature $\left(>550^{\circ} \mathrm{C}\right)$. Coarse-grained films with inhomogeneous grains sizes and high roughness are generally obtained. For this reason, in spite of their very good MO properties, garnets disks have carrier-to-noise ratios currently limited to values less than $60 \mathrm{~dB}$ [11]. The high temperatures required to form the garnet phase call for the use of special glass disks instead of the less expensive polycarbonates disks. Moreover, the pregroove used to guide of the head of the optical drive cannot be pressed as it usually is, i.e. inside a photopolymerizing lacquer deposited before the MO layer. In fact, this layer deteriorates when subjected to temperatures above $200-250^{\circ} \mathrm{C}$.

The cobalt substituted Ba-ferrites and spinel ferrites have received less attention than garnets for MO recording application, because of their lower Faraday rotation and higher optical absorption at short wavelengths. However, new spinel ferrites $\mathrm{M}_{\mathrm{x}} \mathrm{Co}_{\mathrm{y}} \mathrm{Fe}_{3-\mathrm{x}-\mathrm{y}} \mathrm{O}_{4+\mathrm{d}}(\mathrm{M}=$ transition metal cation exhibiting several valence states), called mixed valence defect ferrites, have recently been found advantageous $\mathrm{MO}$ properties and microstructural characteristics [12][13].

In these, the sputtering of mixed oxide targets generates spinel ferrite thin films on glass substrates with no post-treatment. These films are made of very small crystallites of generally less than $100 \mathrm{~nm}$ with a roughness close to $1 \mathrm{~nm}$. But they have low coercivity $(\mathrm{Hc}<1500 \mathrm{Oe})$ and low remanent Faraday rotation $(0.3 \mathrm{deg} / \mu \mathrm{m}$ for $780 \mathrm{~nm}$ wavelength). Annealing treatments carried out below $450^{\circ} \mathrm{C}$ enhance these properties because of the creation of a stress and a short-range order inside the films. The improvement of the properties is especially well correlated with the oxidation phenomena occuring during the heat treatments in air [12]. Coercivities higher than $6000 \mathrm{Oe}$ and high Faraday rotations can then be obtained, especially for the blue wavelengths (fig. 1), while the microstructure (crystallite size about 20-30 nm) and the roughness (Ra \# 2-3 nm) remain satisfactory (fig. 2). Magnetic domains of regular shape have been observed, after writing with a $780 \mathrm{~nm}$ laser diode on a simple structure (glass disk $(1.2 \mathrm{~mm}) / \mathrm{Co}-\mathrm{Mn}$ mixed valence defect ferrite $(360 \mathrm{~nm}) /$ Al reflector $(32 \mathrm{~nm})$ ) (fig. 3). This confirms the advantageous microstructural characteristics of spinel-ferrite thin films.

Mixed valence defect spinel ferrites do seem to have several assets for future MO recording with blue wavelengths. But the drawbacks of these materials are their poor writing sensitivity, because of their high Curie temperature $\left(>300^{\circ} \mathrm{C}\right)$ and the need for annealing to improve the MO properties. However, temperatures above $300^{\circ} \mathrm{C}$ are withing the reach of a $10 \mathrm{~mW}$ blue laser because of the small size of the spot, which leads to high power density, and because of the sufficiently high absorption coefficients of the spinel ferrites in this spectral range. Also, in contrast to the case of the RE-TM alloys and the Co/ $\mathrm{Pt}$ multilayers, ferrites can be heated to high temperatures $\left(300-500^{\circ} \mathrm{C}\right)$ without oxidation or structural damage. Moreover, to avoid decomposition of the pregrooved polymer lacquer, the preformatting informations can be written on the reflective layer deposited after the annealing treatment of the ferrite film. This can be done by a photolithographic process. An example of the marks written on an $\mathrm{Al}$ reflector layer is given in figure 4 . The same type of method can of course be used in making $\mathrm{MO}$ media with garnets.

Other possible ways of getting around the specific problems encountered with the ferrimagnetic oxides are currently being studied. One of them consists in forming the garnet phase or improving the magnetic properties of the spinel ferrites by applying rapid thermal annealings with halogen lamps [14][15]. Lower roughness and smaller mean crystallite size can thus be obtained for garnets. The specific heating of the MO thin films, especially spinel ferrite films because of their higher optical absorption than that of the substrate, could also make possible to use polycarbonate disks. Particulate MO media have also been proposed [16][17]. Erasable optical tape for use as compact cassettes for TV or archival video memories with extremely large capacity (>1Tbytes), are one of the potential applications for this type of medium. In the latter, the particles would be 


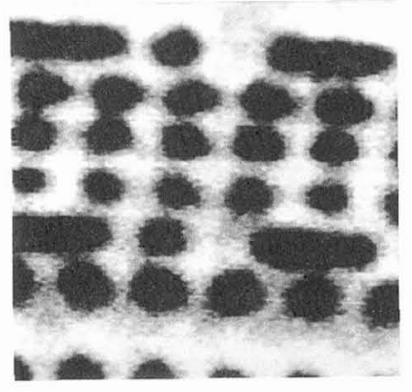

Figure 3: $\mathrm{MO}$ bits in Co-Mn spinel ferrite (square side $=9 \mu \mathrm{m}$ ).

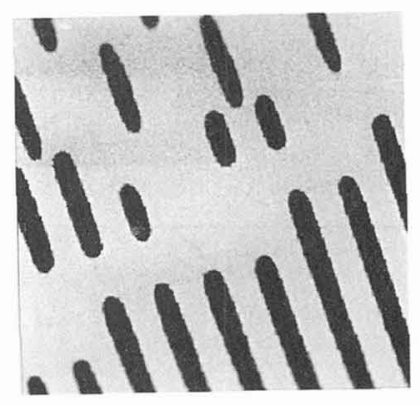

Figure 4: Disk format in $\mathrm{Al}$ reflective layer (square side $=10 \mu \mathrm{m}$ ).

dispersed and embeded in a transparent matrix. The main advantages lie in the fact that particles can be annealed before the medium is coated. The particles must be small with a diameter smaller than the wavelength, so that the data bits can be recorded in small areas of regular shape, which also limits the light diffusion. They must also have the same magnetic and MO properties as defined above for films. Moreover, to minimize the light diffusion intensity and optimize the Faraday rotation of the medium, the binder must have a refractive index close to that of the particles. Particles with such morphology and size can be produced by "chimie douce". However, much work remains to be done to improve the MO properties of the particles and to find organic or hybrid organic-inorganic matrix with the properties defined.

\section{CONCLUSION.}

RE-TM amorphous alloys are currently the best materials for MO recording. However, the progress in laser diodes or frequency doubling systems will make possible to build MO drives with green or blue wavelengths. For these radiations, REMT alloys will be competing with other materials such as metallic multilayers and the very stable ferrites. Of the ferrites, the garnets were the ones most intensively studied; but for the time being, media made with garnets have lower performance than RE-TM media even for blue radiations. However, rapid thermal annealing, particulate MO media or new spinel ferrites, might of fer new possibilities for the use of ferrites.

Because of their advantages, not only in MO recording, but also in magnetic recording applications, new research can now be expected in spinel ferrites. Moreover, the two technologies require certain common properties such as fine microstructure, low roughness and high coercivity films. Therefore, research on these two recording applications will work in combination to increase the chances of obtaining the desired properties. It is also interesting to note that the main qualities and drawbacks of garnets are different from those of spinel ferrites. To combine the advantages of the two types of oxides, the preparation of composite or multilayered materials with spinel ferrites and garnets might thus considered.

For all these reasons, ferrites remain promising for future MO optical recording applications, especially for professional applications in which high life-time and cyclability are required.

\section{References}

[1] Hellman F., Van Dover R.B., Nakahara S., Gyorgy E.M., Phys. Rev. B39, (1989), 10591-10605.

[2] Heitmann H., Hartmann M., Klahn S, Rosenkranz M., Tolle H.J., Willich P., J. Appl. Phys. 61, (1987), 3331-3333.

[3] Greidanus F.J.A.M., KJahn S., Angew. Chem., 101, (1989), 243-249.

[4] Suzuki T., Lin C.J., Bell A.E., IEEE Trans. Mag., 24, (1988), 2452- 2455.

[5] Challener W.A., J. Appl. Phys., 79, (1996), 5693-5695.

[6] Zeper W.B., Van Kestern H.M., Jacobs B.A.J., Spruit J.H.M., Garcia P.F, J. Appl. Phys. 70, (1991), $2264-2271$.

[7] Lin C.J., Do H.V., IEEE Trans. Magn. 26, (1990), 1700-1702.

[8] Krishnan R., Nyvit M., Prosser V., Seddat M., Smetana Z., Tessier M., Visnovsky S., J. Magn. Magn. Mat. 148, (1995), 283-284.

[9] Krishnan R., Lassri H., Seddat M., Porte M., Tessier M., Appl. Phys. Lett. 64, (1994), 2312-2314.

[10] Lin C.J., High density digital recording (NATO-ASI, vol. E229, ed. K.H.J. Buschow, G. J. Long, F. Grandjean, Kluwer Academic Publishers, 1993) pp. 461-481.

[11] Eppler W.R., Kryder M.H., J. Phys. Chem. Solids 56, (1995), 1479-1490.

[12] Bouet L., Tailhades Ph., Rousset A., J. Mag. Mag. Mater. 153, (1996), 389-396.

[13] Bouet L., Tailhades Ph., Rousset A., To be published in J. Mag. Mag. Mater. (Proceedings of the MRM95 Conference)

[14] Suzuki T, J. Appl. Phys. 69, (1991), 4756-4760.

[15] Bouet L., Presmanes L., Tailhades Ph., Rousset A., Legros R., To be published in J. Magn. Soc. Jpn (Proccedings of MORIS 96, Noordwijkerhout, April 29-May 2, 1996).

[16] Gomi M., Abe M., J. Magn. Soc. Jpn, 15, (1991), 227-230.

[17] A be M., Gomi M., J. Magn. Soc. Jpn, 15, (1991), 259-262. 